\title{
From patient to person
}

the need for an 'HIV trajectories' perspective in the delivery of prevention of mother-tochild-transmission services

Hsieh, Amy; Rodrigues, Jessica; Skovdal, Morten; Melillo, Sara; Walker, Damilola;

Community Engagement Working Group of the Interagency Task Team on the Prevention and Treatment of HIV Infection in Pregnant Women, Mothers and Childr

Published in:

AIDS

DOI:

10.1097/QAD.0000000000000341

Publication date:

2014

Document version

Peer reviewed version

Citation for published version (APA):

Hsieh, A., Rodrigues, J., Skovdal, M., Melillo, S., Walker, D., \& Community Engagement Working Group of the Interagency Task Team on the Prevention and Treatment of HIV Infection in Pregnant Women, M. A. C. (2014). From patient to person: the need for an 'HIV trajectories' perspective in the delivery of prevention of mother-tochild-transmission services. AIDS, 28(Suppl 3), S399-S409. https://doi.org/10.1097/QAD.0000000000000341 


\title{
From patient to person: the need for an 'HIV trajectories' perspective in the delivery of prevention of mother-to-child-transmission services
}

\author{
Amy Hsieh $^{a}$, Jessica Rodrigues ${ }^{b}$, Morten Skovdal ${ }^{c, d}$, Sara Melillo ${ }^{e}$, \\ Damilola Walker ${ }^{\mathrm{f}}$, Community Engagement Working Group of the \\ Interagency Task Team on the Prevention and Treatment of HIV \\ Infection in Pregnant Women, Mothers and Children
}

\begin{abstract}
Accelerated efforts to end vertical HIV transmission have resulted in a $52 \%$ decrease in new infections among children since 2001. However, current approaches to prevent mother-to-child-transmission (PMTCT) assume a linearity and universality. These insufficiently guide clinicians and programmes toward interventions that comprehensively address the varying and changing needs of clients. This results in high levels of loss-to-follow-up at each step of the PMTCT cascade. Current PMTCT approaches must be adapted to respond to the different and complex realities of women, children and families affected by HIV.

Drawing on the concept of an 'HIV trajectories,' we screened peer-reviewed literature for promising PMTCT approaches and selected 13 articles for qualitative review when the described intervention involved more than a biomedical approach to PMTCT and mother-child HIV treatment and care. Our qualitative analysis revealed that interventions which integrated elements of the 'HIV trajectories' perspective and built on people living with HIV support/network, community health worker, primary healthcare and early childhood development platforms were successful because they recognized that HIV is an illness, experienced, moderated and managed by numerous factors beyond biomedical interventions alone.

On the basis of this review, we call for the adoption of an 'HIV trajectories' perspective that can help assess the comprehensiveness of care provided to women, children and families affected by HIV and can inform the planning and delivery of HIV and related services so that they more adequately respond to the varying needs of clients on different 'HIV trajectories'.

(c) 2014 Wolters Kluwer Health | Lippincott Williams \& Wilkins
\end{abstract}

AIDS 2014, 28 (Suppl 3):S399-S409

Keywords: child health, community, community engagement, health service delivery, HIV trajectories, life course, PMTCT

\section{Introduction}

Scale-up of services to end the vertical HIV transmission has resulted in a $52 \%$ decrease in the number of new infections among children since 2001 [1]. However, the current epidemic response is failing children living with HIV. In 2012, early infant diagnosis (EID) coverage remained low at 39\% in low-income and middle-income countries, and children diagnosed with HIV were only half as likely as adults living with HIV to access to

\footnotetext{
${ }^{\mathrm{a}}$ Global Network of People Living with HIV, New York, USA, ${ }^{\mathrm{b}}$ UNICEF, New York, USA, ${ }^{\mathrm{C}}$ Department of Public Health, University of Copenhagen, Copenhagen, Denmark, 'Department of Programme Policy and Quality, Save the Children, London, UK, ${ }^{\mathrm{e}}$ Creative Associates International, Washington DC, USA, and ${ }^{\mathrm{f} E l i z a b e t h}$ Glaser Pediatric AIDS Foundation, Washington DC, USA. Correspondence to Morten Skovdal, Department of Public Health, University of Copenhagen, Øster Farimagsgade 51014 Copenhagen, Denmark.

E-mail: m.skovdal@gmail.com
} 
treatment [1]. Political, programmatic and operational challenges as well as slow uptake of newer paediatric formulations, knowledge and technology contribute to inequities in access to EID and paediatric HIV treatment [2]. Although many recent family-centred HIV interventions, and 'one-stop' service integration, have increased uptake of HIV testing and counselling (HTC) and antiretroviral therapy (ART) adherence, these have not translated into greater clinical gains for children.

The dominant framework for providing HIV services has traditionally been the 'treatment cascade' or 'continuum of care', which is a clinical and preplanned trajectories for the care of people living with HIV (PLHIV) [3]. It has been the predominant modality for measuring the effectiveness of HIV care, with viral suppression as the ultimate marker of success [4-7]. For the prevention of mother-to-child-transmission (PMTCT), the stages include initial antenatal care (ANC) visit; HIV test counselling; HIV test acceptance; receipt of HIV diagnosis; prophylaxis/treatment provision to pregnant woman living with HIV; prophylaxis provision to HIVexposed infant (HEI); EID/testing; final infant diagnosis; and linking infants infected with HIV to HIV care and treatment services ART [8-13].

However, treatment cascades assume a linearity and universality, transcending time and place, without considering that HIV is an illness that is experienced and moderated by numerous factors beyond biomedical interventions [14]. The treatment cascade also fails to adequately guide service providers towards comprehensively responding to the complex social realities that govern the health and service access of families affected by HIV. As a consequence, loss-to-follow-up (LTFU) remains a serious problem across any treatment cascade [4-13,15-21].

Against this background, and taking PMTCT efforts as an example, we review promising PMTCT approaches and explore their ability to consider the temporal and broader social determinants of health for women, children and families affected by HIV. Drawing on these lessons, we argue that an 'HIV trajectories' perspective can enhance existing PMTCT services and link clinical interventions with social protection measures and psychological development.

\section{Revisiting the prevent mother-to-child- transmission cascade}

The PMTCT cascade represents points in time when a client received a service, but does not tell the story of events that resulted in a service being provided or neglected. Client LTFU can be attributed to a range of material, symbolic, relational, psychosocial and institutional factors [22,23].
The 'material context' refers to the different resources (e.g. food, household assets, welfare grants, employment and so on) available to families affected by HIV to sustain health and well being. For example, women who experience economic hardship may struggle to meet transport costs associated with clinic appointments. The 'symbolic context' refers to social representations, ideologies and norms in a society that can increase discrimination or further solidarity and support for care. Partners may prevent women from accessing HIV services due to sexual inequalities, for instance [24].

The 'relational context' refers to the quality of social interactions and relationships that characterize family and community relations, which can impact a clients' access to, uptake and quality of services (e.g. poor ANC service provision or lack of family support upon HIV disclosure may affect whether women and their children return to seek clinical care [16]). The 'psychosocial context' refers to how people experience HIV diagnosis and treatment emotionally, psychosocially and socially. Lastly, the 'institutional context' refers to the availability and quality of local institutions to respond to HIV (e.g. ability of civil society organizations to respond to HIV in collaboration with local health services).

The PMTCT cascade acknowledges the clinical needs of pregnant women living with HIV and their children, but the material, symbolic, relational, psychosocial and institutional factors of their social environments often remain unaddressed. These interlinked vulnerabilities, if unmitigated, negatively impact access and utilization of HIV care and treatment services [25]. Rather than being an exception, disengagement could therefore be viewed as inevitable when dealing with a chronic illness [26].

To reduce the likelihood of prolonged disengagement, any model of care provision must recognize and incorporate a client's life course into decisions as to when and how care will be received. Integrating the PMTCT cascade with the 'HIV trajectories' perspective, which highlights vulnerabilities along a client's life course, may identify critical points for intervention that may lead to better health outcomes.

\section{Conceptual framework: towards an 'HIV trajectories' perspective}

The concept of the 'illness trajectories' was first described by Strauss and Corbin $[27,28]$. It asserts that individuals, both those directly and indirectly affected by chronic illness, will experience and respond to an illness differently over time as social and physiological manifestations of the disease change [27,28]. The changing HIV course results from the interactions between clinical disease progression, coinfections and responsiveness to HIV treatment, as well as a web of environmental factors that influence PLHIV [22]. Although HIV treatment cascades, including PMTCT, do include a timeframe and 
a physiological trajectories, it assumes that clients neatly fit into the cascade.

An 'HIV trajectories' perspective differs from the often used socio-ecological perspective in that it evolves as clients' circumstances change and encourages identification of key moments of change that bring people onto different life course trajectories. The temporal and change components of the 'HIV trajectories' urge us to consider the interaction between clinic, patient, family and community-centred HIV care models. Figure 1 serves as a lens to review and assess the comprehensiveness of PMTCT services and care provided to women, children and families affected by HIV.

\section{Enhancing the prevention of mother-to- child-transmission cascade with the 'HIV

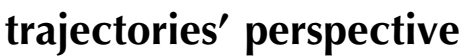

\section{Methods}

Using the lens of the 'HIV trajectories' perspective, we assess promising approaches to more comprehensive service provision for families living with and affected by
HIV. In late 2013, we conducted a literature review using five databases (PubMed, Plosmed, Medline, Cochrane and Google Scholar). We searched a combination of these terms: 'HIV', 'PMTCT', 'community-based' and 'HIVinfected', 'HIV-exposed', 'pregnant women' 'infants' and 'children.' The search was limited to studies carried out in sub-Saharan Africa published in English from January 2000 (when PMTCT became widely available regionally) to January 2014. In all studies, participants included pregnant, breastfeeding women and/or mothers living with HIV and their children. We reviewed studies that involved more than a biomedical approach to providing care for target populations. For all studies, inclusion criteria were analysis of factors related to maternal and/or infant initiation of ARV prophylaxis; maternal and/or infant initiation of ART; uptake of infant testing for HIV and retention of women and/or children living with HIV in care. Studies that examined purely clinical interventions and opinion pieces were excluded.

\section{Results}

The search generated a total of 749 citations. Forty-five articles were selected for further review and 13 were included in this analysis. Titles and abstracts were reviewed and a full screening was performed according to the predetermined inclusion criteria.

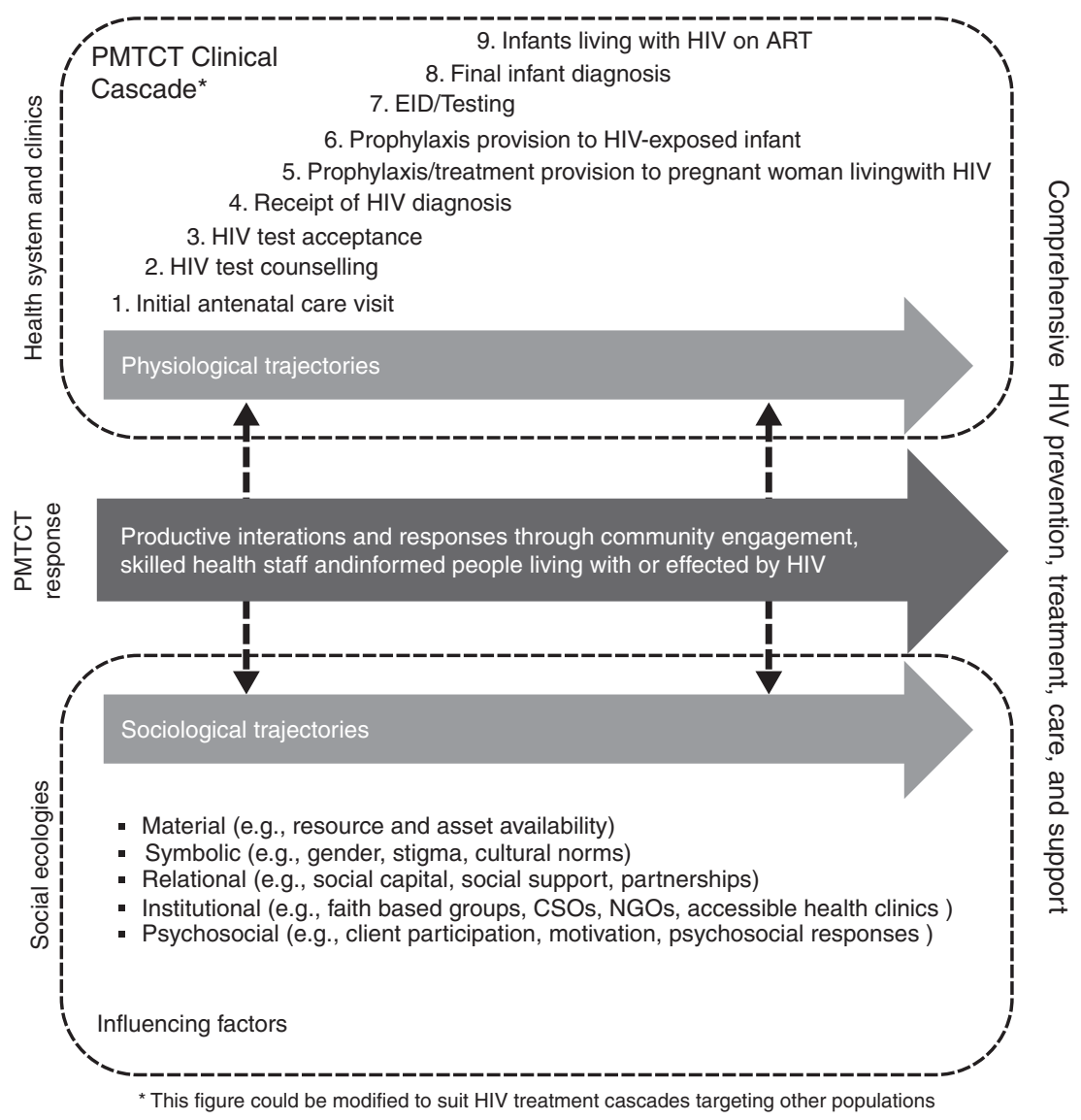

Fig. 1. 'HIV Trajectories' model. 
Outlined below and summarized in Table 1 are interventions that addressed the multiple dimensions of the 'HIV trajectories' perspective in novel ways. These were grouped into four categories of interventions that integrated elements of the 'HIV trajectories' perspective into the PLHIV support/network platform; community health platform; primary healthcare platform [family-centred care (FCC)] and early childhood care and development (ECD) platform. We analysed the core components and qualitative outcomes of the approach when applied in a particular setting to determine which dimensions of the 'HIV trajectories' perspectives were addressed.

Interventions that integrate elements of the trajectories perspective through the people living with HIV support platform

Community-based support groups are popular regionally, affording PLHIV primary roles in the leadership, facilitation and management of such groups. Although there are few studies demonstrating a direct link between community groups and child HIV outcomes, some studies suggest that they create an enabling environment for improved PMTCT outcomes.

A mixed methods assessment demonstrated the effectiveness of a 3-year mentor mother (M2M) program in Bulawayo, Zimbabwe. The intervention linked pregnant women living with HIV with trained mentors also living with HIV who provided information on HIV and sexually transmitted infection prevention and care, birth planning, infant feeding counselling, psychosocial support and ART adherence counselling at the community and household levels. Mothers in this intervention were twice as likely to return for EID, compared with those who had not enrolled in M2M (99.2 versus $48.6 \%, P<0.0005)$ and when tested at 6-8 weeks were more likely to have obtained the result of that test (99.2 versus $73.3 \%, P<0.0005)$ [29].

Although community ART groups (CAG) established in Tete Province in Mozambique did not include children, $61 \%$ of participants in a recent assessment were women of reproductive age and $89 \%$ of pregnant CAG members received PMTCT regimens [30]. This platform could provide a unique opportunity to create demand for HTC and treatment among women of reproductive age and their children. Previous evaluations show that CAGs improve ART adherence through a combination of social support and information sharing [31]. These benefits could have a similar effect on the health-seeking behaviour of pregnant women and mothers living with HIV, by improving retention in ANC, postnatal care and under-five consultations.

Interventions that integrate elements of the trajectories perspective through the community health worker (CHW) platform for HIV outcomes

$\mathrm{CHW}$ programmes are well positioned to bridge clinical and community-based care for HIV-affected women and children. In numerous settings, CHWs, lay counsellors or expert clients with basic training are engaged to provide ANC education and ART adherence counselling, encourage facility deliveries, trace mothers who miss clinical appointments and offer postnatal support in the home. Studies included suggest that using $\mathrm{CHWs}$ to deliver health services produces positive health outcomes related to HIV mortality and viral suppression and to HIV testing rates among infants. In a study that compared the standard of care to CHW home visits from pregnancy to 6 months postpartum for women living with HIV and their children in Cape Town, South Africa, beneficiaries were more likely to obtain key PMTCT services, avoid birth-related medical complications and have infants with healthy height-for-age measurements [32]. A similar intervention in Malawi demonstrated that CHWs could create an effective continuity of care that minimizes LTFU between PMTCT, EID and paediatric HIV services, increasing the proportion of eligible infants initiated on ART from 34.4\% preintervention to $76.7 \%$ [33]. A systematic review of community-based ART delivery showed comparable results to facility-based care, when assessing attrition rates, provider costs and social outcomes [31].

Bringing care to the clients' doorsteps has the potential to ameliorate the material, psychosocial and relational barriers to care. These include reducing transport and other costs associated with going to a health facility, increasing social capital and support, while providing counselling to improve adherence and strengthening linkages to other community resources through referrals. CHWs can also provide key insights into the psychosocial issues that affect clients' adherence or overall well being, and serve as an important bridge between service providers and clients [34].

Interventions that integrate elements of the trajectories perspective through the primary healthcare (family-centred care) platform

There is a widespread recognition of the benefits of reorganizing facility-based services around families' needs as a unit while improving PMTCT and maternal, newborn and child health outcomes [35-40]. FCC approaches have the potential to address the fault lines in the PMTCT cascade by consolidating services for the entire family and tackling the more pervasive socioecological barriers to sustained care. The key FCC programme elements include integrated service provision, fast-tracking families that attend the facility with children, creating child-friendly spaces, EID, providerinitiated testing and strengthening referrals to livelihood projects and education support for children. Familycentred services include [41]:

(1) Maternal and paediatric HIV diagnosis;

(2) Antiretroviral prophylaxis

(3) Cotrimoxazole prophylaxis 


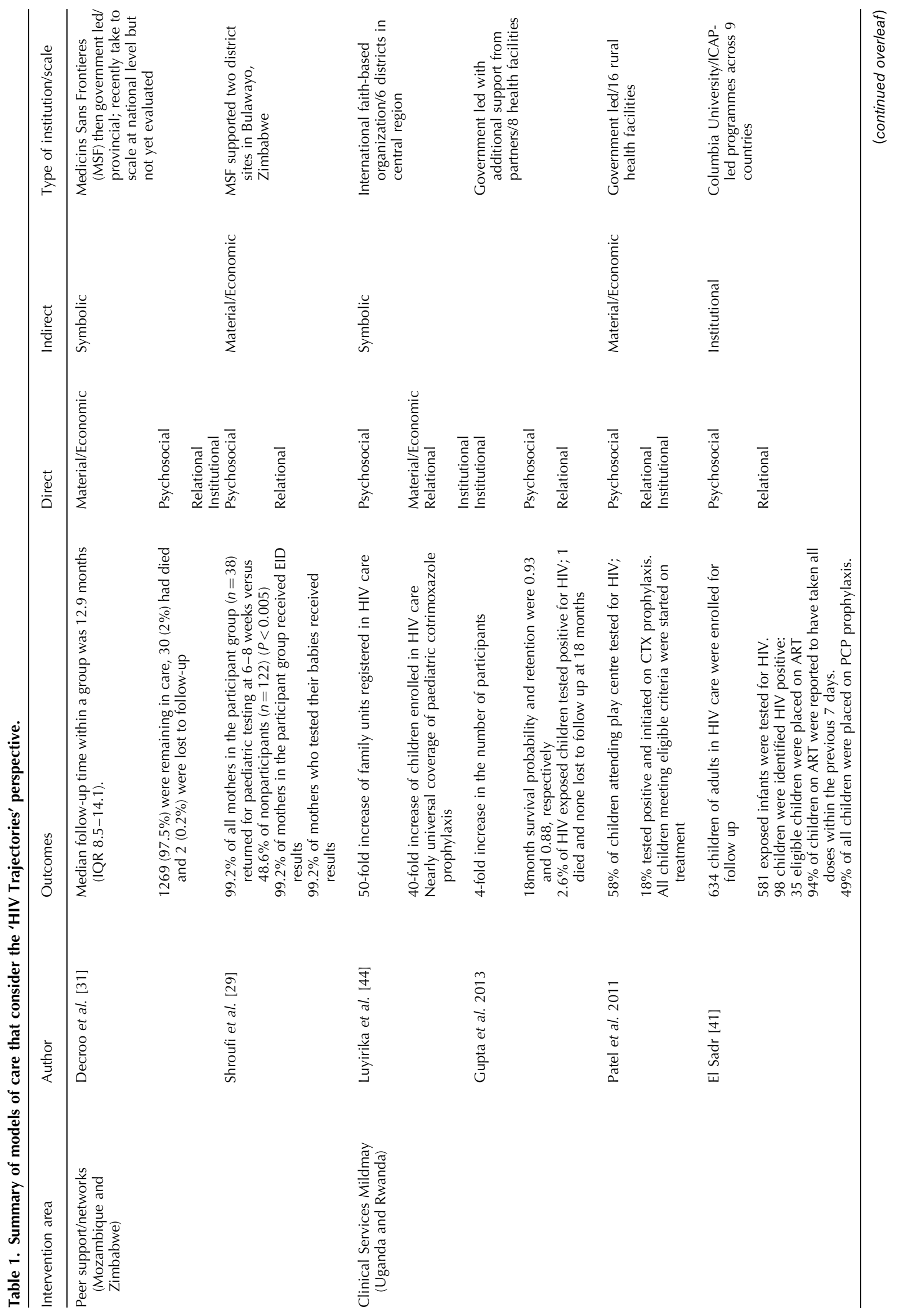




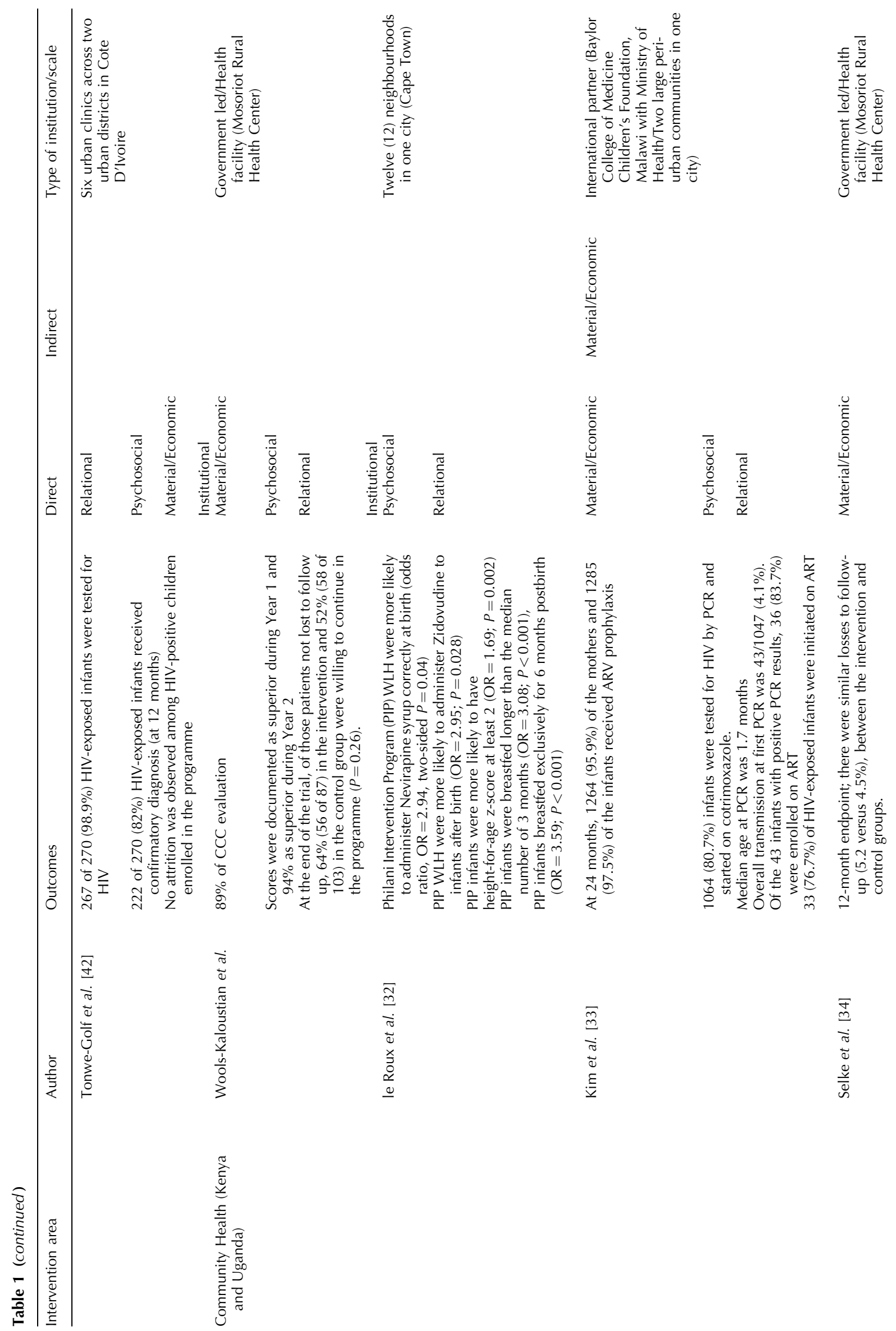


$\overline{0}$
$\frac{\pi}{2}$
0
0
$\frac{1}{0}$
$\frac{0}{0}$
$\frac{0}{60}$
0
0
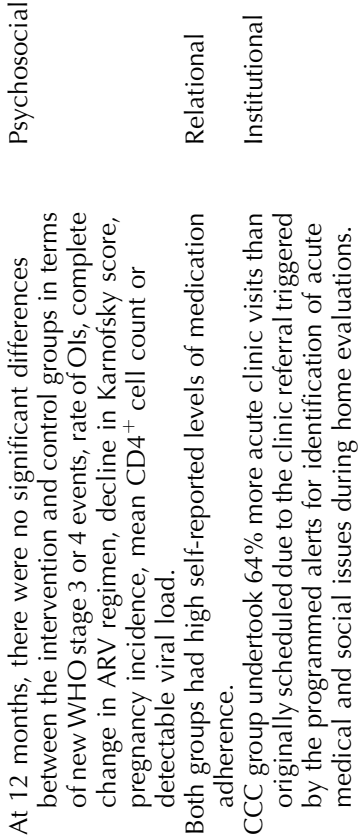

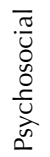

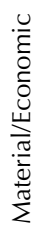

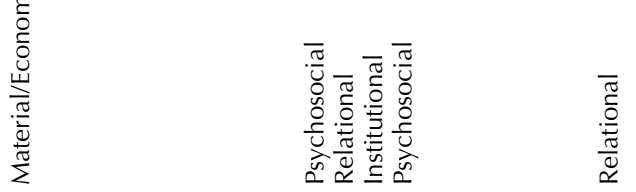

$\frac{\pi}{0}$
$\frac{0}{2}$
$\frac{\pi}{2}$
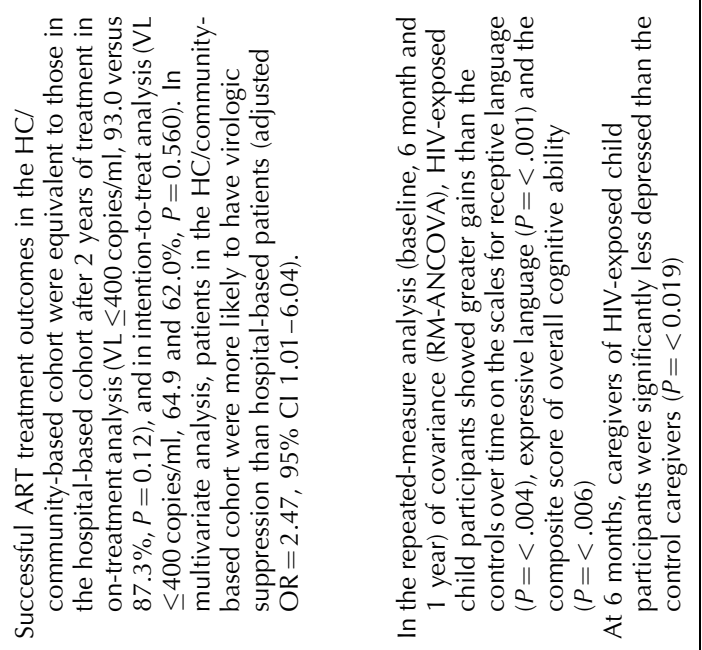

$\frac{N}{2}$
a
$\frac{0}{0}$
$\frac{0}{2}$
$\frac{0}{2}$

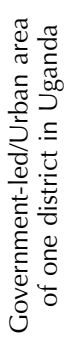

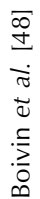

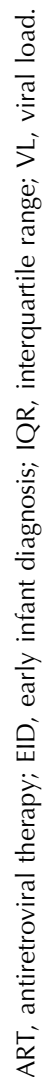

Copyright ( ) Lippincott Williams \& Wilkins. Unauthorized reproduction of this article is prohibited. 
(4) Long-term ART for an entire family

(5) Opportunistic infections;

(6) Infant feeding;

(7) Nutritional support;

(8) Sick and well child care;

(9) Immunizations, growth and monitoring;

(10) Malaria prevention and treatment;

(11) Tuberculosis interventions including isoniazid preventive therapy;

(12) Counselling and psychosocial support;

(13) Referrals to social and economic assistance;

(14) Early childhood development interventions; and

(15) Child-friendly spaces.

This comprehensive package of services addresses the myriad of environmental factors highlighted by the 'HIV trajectories' perspective.

Outcomes of FCC programmes from Uganda, Cote D'Ivoire and DRC support this approach [42-44]. In Uganda, an FCC health centre experienced a 50-fold increase in the number of families registered in HIV care, a 40-fold increase in the number of children enrolled and nearly universal coverage of paediatric cotrimoxazole (CTX) prophylaxis [41]. Equally impressive increases in uptake of EID testing (70\%) and CTX prophylaxis were also reported by the MTCT Plus initiative in Uganda [35]. Uptake of CTX prophylaxis was associated with several benefits for HEI and infants diagnosed with HIV: $46 \%$ reduction in mortality; $30-70 \%$ lower incidence of malaria, diarrhoea and hospitalization; and a $63 \%$ reduction in mortality among HIV-negative children when their parents living with HIV received CTX [35]. Although encouraging, outcomes experienced by children under these various interventions beyond the completion of EID could be strengthened [45].

Interventions that integrate elements of the trajectories perspective through the early childhood care and development platform

Merging community-based ECD programmes and HIV services to enhance services for children has gained traction recently. In Zimbabwe, an evaluation reported that $58.8 \%$ of children were tested for HIV, and all identified as HIV-positive were initiated on CTX and referred into care and treatment through this model [46].

Integrating a wide array of health, ECD and psychosocial services at one site is less costly for clients and can reframe relationships between caregivers, children and staff. It provides more nuanced insights into the child's psychological development, enabling both health staff and caregivers to adjust care according to a child's changing needs over time. Offering ECD training for caregivers in Uganda improved the language skills and cognitive ability of children exposed to and living with HIV and long-term retention of children enrolled in treatment was encouraging at a site that combined psychosocial support and treatment literacy into healthcare $[47,48]$. At 6 months, caregivers of HIV-exposed children participating in the intervention were significantly less depressed than the control caregivers, irrespective of caregiver category $(\mathrm{F}[1]=6.14, P=0.019$, observed power $=0.69)$. Among children participating, the group versus control interaction indicated significant outcome differences over time for gross motor skills $(P \leq 0.023)$, receptive language $(P \leq 0.04)$, expressive language $(P \leq 0.01)$ and early learning composite scores $(P \leq 0.06)$. This intervention addresses many of the 'HIV trajectories' dimensions, as it recognizes the interdependence between health outcomes and the emotional and cognitive stages of child development.

\section{Discussion}

Receiving an HIV diagnosis is a life-changing, seismic event for a pregnant woman, with implications beyond her clinical experience. Interventions that address this reality are more likely to succeed in delivering improved outcomes for mothers and children living with HIV. The interventions we highlight above depart from prevailing approaches by more fully addressing the varied life experiences of PLHIV and their families.

To varying degrees, all the approaches reduce the economic burden on clients and make care-seeking more convenient. These approaches also consider the profound influence that relationships have on individual care-seeking behaviour for people living with a chronic illness. Whether it is through improving communication between providers and clients by using CHWs or peers to serve as liaisons, these models put relationship building at the forefront. It is also through communication and dialogue that symbolic barriers (e.g. stigma) can be challenged, and where therapeutic identities can be renegotiated $[49,50]$. CHWs, expert clients and lay cadres can help to overcome institutional barriers to care caused by high volumes and severe human resource shortages in resource-limited settings.

These studies illustrate the centrality of the psychosocial dimensions of clients' experiences and health-seeking behaviours. Generally, these models foster increased autonomy and self-reliance among clients and communities, directly enabling adherence and information sharing, and influencing behaviours within the community. This can help to counter a client's reluctance to continue treatment when feeling healthy or better after a prolonged period on treatment. Additional communityoriented models can help to dissipate stigma, increase access to social and economic assistance, and reduce mental or psychological distress [51-53]. 
Despite growing evidence on the acceptability and operational feasibility of approaches that acknowledge the 'HIV trajectories' perspective, pilot efforts predominate and broader scale-up and mainstreaming has not occurred on a national or sub-national scale. Fundamental changes are required to budgeting, policymaking, programmes and service delivery to reduce the barriers to scale-up of more holistic approaches to health and HIV care for women, children and families affected by HIV.

For policymakers and donors, increased coordination between the line ministries responsible for health, social services, child protection, social welfare, women's issues and education should be at the heart of any enduring move towards client-responsive services. This will help overcome the historical silos of HIV programmes that contribute to a disjointed response to the needs of children affected by HIV.

At the programmatic level, and responding directly to the maturity and dynamics of the local epidemic, the local economy and sociocultural context, programme managers must determine the optimal mix of interventions that meet the needs of mothers and their children at each stage of their life course and their 'HIV trajectories'. For instance, internal and cross-border migration is a specific programmatic challenge with few solutions in the contexts where there is a high degree of mobility during the perinatal period. Finally, as service and technological innovations arise, concerted effort will be needed to study how they can be adapted to meet the specific needs across the 'HIV trajectories' for mothers, children and families, starting from pregnancy onwards.

If fully operationalized at the service-delivery level, providing care that responds to the clients' and families' place along their 'HIV trajectories' demands a paradigm shift in the delivery of health services. First, an enabling environment that devolves decision-making and planning to the lowest levels possible must be in place. In addition, care systems must be organized and coordinated to fully leverage and empower diverse disciplines (e.g. medicine, nursing, child life, welfare, social work, psychology, legal and so on) to deliver tailored and client-responsive services. Supports to enable task-shifting to nurses, CHW and lay cadres (e.g. patient advocates, escorts) will also be an important enabling element [7]. However, taskshifting must be carefully weighed and supported with an eye on quality. Task-shifting can exacerbate health system fragility by increasing the workload of nonphysician health cadres, with failure to address poor working conditions and pay leading to turnover [54]. Effective CHW and nurse training and mentorship are critical to ensuring that patients receive high-quality care and effective management of complex clinical complications.

This review also highlights many outstanding questions to be explored as the HIV response matures. More studies are needed to evaluate the effectiveness of integrated approaches and trajectories-sensitive interventions targeted at children affected by HIV, including their ECD needs. Longitudinal follow-up through adolescence will be required, as their needs will evolve over time. Approaches to planning and deployment for the current human resource workforce will need to be carefully considered. The full complement and skills mix required to enable tailored care is unclear, and will need to be carefully documented. This review could not answer the issue of costing and cost-effectiveness of the different interventions.

\section{Conclusion}

Many of the models discussed have important elements that consider the 'HIV trajectories' of women, children and families living with and affected by HIV. It is time that we join essential biomedical and community-based interventions into a unified, adaptable and scalable model. There is potential to build on the strengths of ECD platforms to offer and monitor essential health services for children affected by and living with HIV. Similarly, PMTCT programmes can learn from and incorporate community-based approaches as a standard component of care, rather than an add-on.

The implication of the 'HIV trajectories' perspective is that comprehensive HIV prevention, treatment, care and support cannot be achieved if we fail to place the client in context. Recognition of client trajectories has the potential to offer clients, caregivers and service providers a better understanding of what contributes to HIV treatment success. Such recognition can empower everyone involved to feel a sense of control and support their efforts to cope with the demands of the illness [55].

However, caution needs to be taken not to boldly assign clients onto a 'common' trajectories. Life circumstances can, at any point, take clients down different trajectories, requiring us to regularly review clients' situations. We need the tools and approaches to address this.

A radically different approach, as proposed with the 'HIV trajectories' perspective, that links social protection measures, psychological development and clinical care is required to reduce vertical transmission of HIV, maternal and infant mortality, provide universal coverage of paediatric ART and address the particularly stubborn areas where we see low uptake and retention in services [56]. This will better ensure that children and their families receive care that reflects the complex realities of living with a chronic illness and, in turn, accelerate the achievement of the Global Plan targets by 2015 [57]. 


\section{Acknowledgements}

\section{Conflicts of interest}

A.H., J.R. and M.S. conceived the review, contributed to the literature review, drafted portions and edited the manuscript. J.R. compiled the first draft of the manuscript. D.W. contributed to the literature review, drafted portions and edited the manuscript. S.M. drafted portions and edited the manuscript. The manuscript was revised critically for intellectual content by all the coauthors, who all read and approved the final manuscript. The authors reserve the right to add Paul Young to the list of authors.

Any opinions, findings, conclusions or recommendations expressed in this material are those of the authors and do not necessarily reflect the official positions of the Creative Associates International, GNP+, IATT, Elizabeth Glaser Pediatric AIDS Foundation, Save the Children, UNICEF or the U.S. government, including the U.S. Centers for Disease Control and Prevention.

The authors did not receive any financial compensation for their contributions to this article.

\section{References}

1. UNAIDS. 2013 progress report on the global plan. UNAIDS, UNICEF and WHO. http://www.unaids.org/en/media/unaids/ contentassets/documents/unaidspublication/2013/20131129_ stocktaking_report_children_aids_en.pdf. Accessed December 3, 2013.

2. WHO and UNICEF. For improved pediatric HIV diagnosis, care and treatment in high HIV prevalence countries and regions. Pediatric Advocacy Toolkit. December 2011. http://www.who. int/hiv/pub/pediatric toolkit2011/en/ [Accessed 26 March 2014].

3. Gardner EM, McLees MP, Steiner JF, del Rio C, Burman WJ. The spectrum of engagement in HIV care and its relevance to testand-treat strategies for prevention of HIV infection. Clin Infect Dis 2011; 52:793-800.

4. Rosen S, Fox MP. Retention in HIV care between testing and treatment in sub-Saharan Africa: a systematic review. PLOS Med 2011; 8:e1001056.

5. Mugglin C, Estill J, Wandeler G, Bender N, Egger M, Gsponer T, et al. Loss to programme between HIV diagnosis and initiation of antiretroviral therapy in sub-Saharan Africa: systematic review and meta-analysis. Trop Med Int Health 2012; 17:1509-1520.

6. Kranzer K, Govindasamy D, Ford N, Johnson V, Lawn SD. Quantifying and addressing losses along the continuum of care for people living with HIV infection in sub-Saharan Africa: a systematic review. I Int AIDS SOC 2012; 15:17383.

7. WHO. Consolidated guidelines on general HIV care and the use of antiretroviral drugs for treating and preventing HIV infection: recommendations for a public health approach. WHO, Geneva; 2013.

8. Mugglin C, Wandeler G, Estill J, Egger M, Bender N, Davies M Keiser O. Retention in care of HIV-infected children from HIV test to start of antiretroviral therapy: systematic review. PLOS One 2013; 8:e56446.

9. Car LT, Brusamento $\mathrm{S}$, Elmoniry $\mathrm{H}$, van Velthoven $\mathrm{MH}$, Pape UJ, Welch $\mathrm{V}$, et al. The uptake of integrated perinatal prevention of mother-to-child HIV transmission programs in low- and middle-income countries: a systematic review. PLOS One 2013; 8:e56550.

10. Perez F, Mukotekwa T, Miller A, Orne-Gliemann J, Glenshaw M, Chitsike I, Dabis F. Implementing a rural programme of prevention of mother-to-child transmission of HIV in Zimbabwe: first 18 months of experience. Trop Med Int Health 2004; 9:774-783.
11. Rutta E, Gongo R, Mwansasu A, Mutasingwa D, Rwegasira V, Kishumbu $S$, et al. Prevention of mother-to-child transmission of HIV in a refugee camp setting in Tanzania. Glob Public Health 2008; 3:62-76.

12. Shetty AK, Marangwanda C, Stranix-Chibanda L, Chandisarewa W, Chirapa E, Mahomva A, et al. The feasibility of preventing mother-to-child transmission of HIV using peer counselors in Zimbabwe. AIDS Res Ther 2008; 5:1-8.

13. Magoni M, Okong P, Bassani L, Kituka NP, Onyango S, Giuliano $M$. Implementation of a programme for the prevention of mother-to-child transmission of HIV in a Ugandan hospital over five years: challenges, improvements and lessons learned. Int / STD AIDS 2007; 18:109-113.

14. Lock M, Nguyen V-K. An anthropology of biomedicine. Malden, MA: Wiley-Blackwell; 2010.

15. McNairy ML, Lamb MR, Carter RJ, Fayorsey R, Tene G, Mutabazi $V$, et al., Identifying optimal models of HIV care and treatment in sub-Saharan Africa Consortium. Retention of HIV-infected children on antiretroviral treatment in HIV care and treatment programs in Kenya, Mozambique, Rwanda, and Tanzania. I Acquir Immune Defic Syndr 2013; 62:e70-e81.

16. Sibanda EL, Weller IVD, Hakim JG, Cowan FM. The magnitude of loss to follow-up of HIV-exposed infants along the prevention of mother-to-child HIV transmission continuum of care: a systematic review and meta-analysis. AIDS 2013; 27:27872797.

17. Shargie MB, Eek F, Abaychew A. Prophylactic treatment uptake and compliance with recommended follow up among HIV exposed infants: a retrospective study in Addis Ababa, Ethiopia. BMC Res Notes 2011; 4:563-1563.

18. Anoje C, Aiyenigba B, Suzuki C, Badru T, Akpoigbe K, Odo M, et al. Reducing mother-to-child transmission of HIV: findings from an early infant diagnosis program in south-south region of Nigeria. BMC Public Health 2012; 184:12.

19. Hassan AS, Sakwa EM, Nabwera HM, Taegtmeyer MM, Kimutai RM, Sanders EJ, et al. Dynamics and constraints of early infant diagnosis of HIV infection in rural Kenya. AIDS Behav 2012; 16:5-12.

20. Nuwagaba-Biribonwoha $\mathrm{H}$, Werq-Semo B, Abdallah A, Cunningham A, Gamaliel JG, Mtunga S, et al. Introducing a multisite program for early diagnosis of HIV infection among HIVexposed infants in Tanzania. BMC Pediatr 2010; 10:44.

21. Violari A, Cotton MF, Gibb DM, Babiker AG, Steyn J, Madhi SA, et al. Early antiretroviral therapy and mortality among HIVinfected infants. N Engl / Med 2008; 359:2233-2244.

22. Skovdal M, Campbell C, Nhongo K, Nyamukapa C, Gregson S. Contextual and psychosocial influences on ART adherence in rural Zimbabwe: towards a systematic framework for programme planners. Int J Health Plann Manage 2011; 26:296-318.

23. Campbell C, Cornish F. Towards a 'fourth generation' of approaches to HIV/AIDS management: creating contexts for effective community mobilisation. AIDS Care 2008; 22 (Suppl 2):1569-1579.

24. Skovdal M, Campbell C, Madanhire C, Nyamukapa C, Gregson $S$. When masculinity interferes with women's treatment of HIV infection: a qualitative study about adherence to antiretroviral therapy in Zimbabwe. J Int AIDS SOC 2011; 14l:29.

25. Duff P, Kipp W, Wild TC, Rubaale T, Okech-Ojony J. Barriers to accessing highly active antiretroviral therapy by HIV-positive women attending an antenatal clinic in a regional hospital in western Uganda. I Int AIDS SOC 2010; 13:37.

26. Ware NC, Wyatt MA, Geng EH, Kaaya SF, Agbaji OO, Muyindike WR, et al. Toward an understanding of disengagement from HIV treatment and care in sub- Saharan Africa: a qualitative study. PLoS Med 2013; 10:e1001369.

27. Strauss AL. Chronic illness and the quality of life. St. Louis, MO: The C. V. Mosby Company; 1984.

28. Corbin JM, Strauss A. Unending work and care: managing chronic illness at home. San Francisco, CA: Jossey-Bass Publishers; 1988.

29. Shroufi A1, Mafara E, Saint-Sauveur JF, Taziwa F, Viñoles MC. Mother to mother (M2M) peer support for women in prevention of mother to child transmission (PMTCT) programmes: a qualitative study. PLoS One 2013; 8:e64717.

30. Geelhoed D, Decroo T, Dezembro S, Matias H, Lessitala F, Muzila F, et al. Utilization of and barriers to HIV and MCH services among community ART group members and their families in Tete, Mozambique. AlDS Res Treatment 2013; 2013:937456. 
31. Decroo T, Rasschaer F, Telfer B, Remartinez D, Laga M, Ford N. Community-based antiretroviral therapy programs can overcome barriers to retention of patients and decongest health services in sub-Saharan Africa: a systematic review. Int Health 2013; 5:169-179.

32. le Roux IM, Tomlinson M, Harwood JM, O'Connor MJ, Worthman CM, Mbewu $N$, et al. Outcomes of home visits for pregnant mothers and their infants: a cluster randomized controlled trial. AIDS 2013; 27:1461-1471.

33. Kim MH, Ahmed S, Buck WC, Preidis GA, Hosseinipour MC, Bhalakia $A$, et al. The Tingathe programme: a pilot intervention using community health workers to create a continuum of care in the prevention of mother to child transmission of HIV (PMTCT) cascade of services in Malawi. / Int AIDS SOC 2012; 15 (Suppl 2):17389.

34. Selke HM, Kimaiyo S, Sidle JE, Vedanthan R, Tierney WM, Shen $\mathrm{C}$, et al. Task-shifting of antiretroviral delivery from healthcare workers to persons living with HIV/AIDS: clinical outcomes of a community-based program in Kenya. I Acquir Immune Dec Syndr 2010; 55:483-490.

35. Betancourt TS, Abrams EJ, McBain R, Smith Fawzi MC Family-centred approaches to the prevention of mother to child transmission of HIV. J Int AIDS SOC 2010; 13:S2.

36. Rochat TJ, Bland R, Coovadiab $H$, Stein A, Newell M. Towards a family-centered approach to HIV treatment and care for HIV-exposed children, their mothers and their families in poorly resourced settings. Future Virol 2011; 6:687696.

37. Gennaro V, Zeitz P. Embracing a family-centred response to the HIV/AIDS epidemic for the elimination of pediatric AIDS. Glob Public Health 2009; 4:386-401.

38. Tolle MA. A package of primary healthcare services for comprehensive family-centred HIV/AIDS care and treatment programs in low-income settings. Trop Med Int Health 2009; 14:663-672.

39. Chandan $U$, Richter L. Strengthening families through early intervention in high HIV prevalence countries. AIDS Care 2009; 21:76-82.

40. Kiene SM, Stein M, Bateganya M, Wanyenze R, Mayer K, Lule $H$. Provider-initiated HIV testing in healthcare settings: should it include client-centered counselling? / SOC Aspects HIV/AIDS 2009; 6:115-119.

41. Luyirika E, Towle MS, Achan J, Muhangi J, Senyimba C, Lule F, Muhe L. Scaling up paediatric HIV care with an integrated, family-centred approach: an observational case study from Uganda. PLoS One 2013; 8:e69548.

42. El-Sadr WM, Rabkin M, Abrams EJ, Hoos D, Berkman A, Myer L, et al. Successful enrollment of families with HIV disease in the MTCT-plus initiative. Proceedings of the XV International AIDS Conference; 11-16 July, 2004; Bangkok, Thailand.

43. Tonwe-Gold B, Ekouevi DK, Bosse CA, Toure S, Kone M Becquet $\mathrm{R}$, et al. Implementing family-focused HIV care and treatment: the first 2 years' experience of the mother-to-child transmission-plus program in Abidjan, Cote d'Ivoire. Trop Med Int Health 2009; 13:204-212.
44. Yalala S, Roger I, Kabamba J, Mavakala K, Tabala M, Sander P, et al. Family-centered HIV services at a primary healthcare center for women identified as HIV+ during antenatal care. Proceedings of the XVII International AIDS Conference; 3-8 August, 2008; Mexico City.

45. Leeper S, Montague B, Friedman J, Flanigan T. Lessons learned from family-centred models of treatment for children living with HIV: current approaches and future directions. I Int AIDS Soc 2010; 13 (Suppl 2):S3.

46. Patel D, Matyanga P, Nyamundaya T, Chimedza D, Webb K, Engelsmann B. Facilitating HIV testing, care and treatment for orphans and vulnerable children aged five years and younger through community-based early childhood development playcentres in rural Zimbabwe. J Int AIDS SOC 2012; 15 (Suppl 2): 17404.

47. Boivin MJ, Bangirana P, Nakasujja N, Page CF, Shohet C, Givon $\mathrm{D}$, et al. A year-long caregiver training program improves cognition in preschool Ugandan children with human immunodeficiency virus. I Pediatr 2013; 163:1409-1416.

48. Boivin MJ, Bangirana P, Nakasujja N, Page CF, Shohet C, Givon $\mathrm{D}$, et al. A year-long caregiver training program to improve neurocognition in preschool Ugandan HIV-exposed children. J Dev Behav Pediatr 2013; 34:269-278.

49. Campbell C, Skovdal M, Madanhire C, Nyamukapa C, Gregson $S$. We, the AIDS people: through what mechanisms have antiretroviral therapy created a context for ARV users to resist stigma and construct positive identities? Am J Public Health 2011; 101:1004-1010.

50. Mburu G, Ram M, Skovdal M, Bitira D, Hodgson I, Mwai G, et al. Resisting and challenging stigma in Uganda: the role of support groups of people living with HIV. J Int AIDS SOC 2013; 16 (Suppl 2): 18636

51. Zhao G, Li X, Zhao J, Zhang L, Stanton B. Relative importance of various measures of HIV-related stigma in predicting psychological outcomes among children affected by HIV. Commun Mental Health J 2012; 48:275-283.

52. Richter L. The impact of HIV/AIDS on the development of children. In: Pharoach R. editor. A generation at risk? HIV/ AIDS, vulnerable children and security in Southern Africa Cape Town: Institute of Security Studies; 2004. pp. 9-31.

53. Sipsma H, Eloff I, Makin J. Behavior and psychological functioning of young children of HIV-positive mothers in South Africa. AIDS Care 2013; 25:721-725.

54. Kankasa C, Carter RJ, Briggs N, Bulterys M, Chama E, Cooper $E R$, et al. Routine offering of HIV testing to hospitalized pediatric patients at university teaching hospital, Lusaka, Zambia: acceptability and feasibility. I Acquir Immune Defic Syndr 2009; 51:202-208.

55. Zelnick J, O'Donnell M. Expansion of the health workforce and the HIV epidemic. N Engl J Med 2008; 358:1639-1640.

56. Murray SA, Kendall M, Boyd K, Sheikh A. Illness trajectories and palliative care. $B M J$ 2005; 330:1007-1011.

57. UNAIDS. Global plan towards the elimination of new HIV infections among children by 2015 and keeping their mothers alive: 2011-2015.2011. http://www.unaids.org/en/media/unaids/ contentassets/documents/unaidspublication/2011/20110609 jc2137_global-plan-elimination-hiv-children_en.pdf. Accessed December 3, 2013. 